\title{
BMJ Open Investigating the Lowest Threshold of Vascular Benefits from LDL Cholesterol Lowering with a PCSK9 mAb Inhibitor (Alirocumab) in Patients with Stable Cardiovascular Disease (INTENSITY- HIGH): protocol and study rationale for a randomised, open label, parallel group, mechanistic study
}

To cite: Cacciottolo PJ, Kostapanos MS, Hernan Sancho E, et al. Investigating the Lowest Threshold of Vascular Benefits from LDL Cholesterol Lowering with a PCSK9 mAb Inhibitor (Alirocumab) in Patients with Stable Cardiovascular Disease (INTENSITYHIGH): protocol and study rationale for a randomised, open label, parallel group, mechanistic study. BMJ Open 2021;11:e037457. doi:10.1136/ bmjopen-2020-037457

- Prepublication history and supplemental material for this paper is available online. To view these files, please visit the journal online (http://dx.doi org/10.1136/bmjopen-2020037457).

Received 04 February 2020 Revised 04 January 2021 Accepted 26 February 2021

Check for updates

(C) Author(s) (or their employer(s)) 2021. Re-use permitted under CC BY-NC. No commercial re-use. See rights and permissions. Published by BMJ.

For numbered affiliations see end of article.

Correspondence to Dr Paul J Cacciottolo; pjc99@medschl.cam.ac.uk

Paul J Cacciottolo (D) , ${ }^{1}$ Michalis S Kostapanos (D) , ${ }^{2}$ Elena Hernan Sancho, ${ }^{3}$ Holly Pavey, ${ }^{3}$ Fotini Kaloyirou, ${ }^{1}$ Evangelia Vamvaka, ${ }^{1}$ Joanna Helmy, ${ }^{1}$ Annette Hubsch, ${ }^{1}$ Carmel M McEniery, ${ }^{1}$ Ian B Wilkinson, ${ }^{1,3}$ Joseph Cheriyan (D) ${ }^{1,3}$

\section{ABSTRACT}

Introduction Elevated low-density lipoprotein cholesterol (LDL-C) is a strong independent risk predictor of cardiovascular (CV) events, while interventions to reduce it remain the only evidence-based approach to reduce CV morbidity and mortality. Secondary prevention statin trials in combination with ezetimibe and/or proprotein convertase subtilisin/kexin type 9 (PCSK9) inhibitors showed that there is no 'J shaped curve' in LDL-C levels with regard to CV outcomes. The lowest threshold beyond which reduction of LDL-C confers no further $\mathrm{CV}$ benefits has not been identified.

The INTENSITY-HIGH study seeks to explore physiological mechanisms mediating CV benefits of LDL-C lowering by PCSK9 inhibition in patients with established cardiovascular disease (CVD). The study examines the changes in measures of endothelial function and vascular inflammation imaging following intervention with PCSK9 and against standard of care.

Methods and analysis This is a single-centre, randomised, open label, parallel group, mechanistic physiological study. It will include approximately 60 subjects with established CVD, with LDL-C of $<4.1 \mathrm{mmol} / \mathrm{L}$ on high-intensity statins. All eligible participants will undergo 18-fluorodeoxyglucose positron emission tomography/CT (FDG-PET/CT) scanning of the aorta and carotid arteries, as well as baseline endothelial function assessment. Subsequently, they will be randomised on a $1: 1$ basis to either alirocumab $150 \mathrm{mg}$ or ezetimibe $10 \mathrm{mg} /$ day. Repeat FDG-PET/CT scan and vascular assessments will be undertaken after 8 weeks of treatment. Any changes in these parameters will be correlated with changes in lipid levels and systemic inflammation biomarkers.

\section{Strengths and limitations of this study}

- PCSK9-inhibitors are currently licensed in cardiovascular (CV) patients with uncontrolled low-density lipoprotein cholesterol (LDL-C). Their benefits, however, may extend, in such high-risk patients, irrespective of target LDL-C levels. This study assesses the physiological responses to aggressive LDL-C lowering on vascular inflammation and endothelial function in stable patients.

- The use of 18-fluorodeoxyglucose positron emission tomography/CT as a marker of vascular inflammation in an interventional study will provide a mechanistic understanding to explain potential benefits in this population on top of existing evidence-based therapies.

- The relative short duration of the trial means the study is not powered to assess clinical outcome data, but provides a rationale for the extension of current guidelines to treat $\mathrm{CV}$ patients more aggressively in terms of addressing the issue of residual risk.

Ethics and dissemination The study received a favourable opinion from the Wales Research Ethics Committee 4, was registered on clinicaltrials.gov and conformed to International Conference for Harmonisation of Technical Requirements for Registration of Pharmaceuticals for Human Use Good Clinical Practice. The results of this study will be reported through peerreviewed journals and conference presentations. Trial registration number NCT03355027. 


\section{BACKGROUND}

Atherosclerotic cardiovascular disease (CVD) remains a significant cause of morbidity and mortality, despite major advances in its detection and treatment. The link between inflammation and atherosclerosis is firmly established, ${ }^{1}$ and the role of cholesterol in its pathophysiology is well described. ${ }^{2}$

Atherogenesis is a chronic, subclinical inflammatory process in which apolipoprotein(apo)B-containing lipoproteins, inflammatory cells and other connective tissue cells accumulate in the subendothelial space resulting in endothelial dysfunction, vascular inflammation and arterial stiffness. Impaired endothelial function facilitates further accumulation of atherogenic lipoproteins and attracts inflammatory cells (circulating monocytes) in the subendothelial space. There is cross-talk between vascular inflammation and endothelial dysfunction that leads to atherosclerosis, and subsequently to symptomatic CVD and atherothrombotic events secondary to rupture of inflamed lipid-rich unstable plaques.

Low-density lipoprotein (LDL) particles are highly atherogenic. Elevated levels of low-density lipoprotein cholesterol (LDL-C) strongly and independently predict cardiovascular (CV) events. ${ }^{2}$ Statins are first-line evidencebased drugs to reduce CV morbidity and mortality in parallel with their LDL-C lowering capacity. ${ }^{3}$ Various studies have suggested that statins exert additional pleiotropic benefits, including an improvement in endothelial function, anti-inflammatory and anti-oxidant actions, as well as reduced arterial stiffness and atheromatous plaque stabilisation. ${ }^{45}$ It is still debated whether these benefits could be explained solely by LDL-C lowering. We have previously shown that comparable reductions of LDL-C with either simvastatin or ezetimibe resulted in similar improvements in endothelial function by significantly limiting inflammation in patients with rheumatoid arthritis. ${ }^{6}$ This suggests that LDL-C lowering may be a key mechanism by which vascular benefits of lipid modifying treatment are exerted.

Despite aggressive LDL-C lowering with statins, a considerable $\mathrm{CV}$ risk remains both in the primary and secondary prevention settings. This residual risk can be explained, at least partly, by subclinical endothelial dysfunction and concomitant subclinical vascular inflammation. The independent prognostic value of endothelial dysfunction markers for CV events has been well established. ${ }^{7}$ Changes in endothelial function can be detected not only at the site of coronary or carotid artery lesions, but also in peripheral vessels remote from the site of structural abnormalities or occlusive disease. ${ }^{8}$

\section{LDL-C lowering therapies}

Statin trials have proven that CV benefits extend to LDL-C levels below $1.8 \mathrm{mmol} / \mathrm{L}$ in patients with CVD. ${ }^{9}$ Recently, even lower LDL-C levels have been shown to improve outcomes in patients with acute coronary syndromes. ${ }^{1011}$ This has prompted lower LDL-C target recommendations in the 2019 European Society of Cardiology Dyslipidaemia guidelines for very high risk and high risk patients to $<1.4 \mathrm{mmol} / \mathrm{L}$ and $<1.8 \mathrm{mmol} / \mathrm{L}$ respectively. ${ }^{12}$ Despite this, it is still unclear what the lowest threshold of total and LDL-C should be to attain significant reductions in CV risk in patients with established CVD. ${ }^{13}$ Additionally, it is unclear if there is a true LDL-C level beyond which there is no further improvement in endothelial function. Whether endothelial function continues to improve towards very low levels of LDL-C in correlation with changes in systemic and vascular inflammation markers remains to be elucidated.

Proprotein convertase subtilisin/kexin type 9 (PCSK9) inhibitors have emerged as new therapies able to reduce LDL-C to previously unattainable levels. Their main lipid action is LDL-C reduction by blocking the degradation of LDL receptors; a process found to be facilitated by statins. Therefore, PCSK9 inhibition by alirocumab or evolocumab efficiently reduces LDL-C by up to approximately $60 \%$ even on top of pre-existing statin treatment. ${ }^{14} 15$ Post hoc analysis of the Evaluation of Cardiovascular Outcomes After an Acute Coronary Syndrome During Treatment With Alirocumab (ODYSSEY OUTCOMES) trial suggested that additional LDL-C reduction by alirocumab on top of statin treatment is associated with further decreased risk of CV events. ${ }^{15}$ Similarly, the Further Cardiovascular Outcomes Research With PCSK9 Inhibition in Subjects With Elevated Risk (FOURIER) trial showed that evolocumab significantly reduced $\mathrm{CV}$ events compared with placebo in patients with established CVD and LDL-C $\geq 1.8 \mathrm{mmol} / \mathrm{L}$ or non-high-density lipoprotein (HDL) cholesterol $\geq 2.6 \mathrm{mmol} / \mathrm{L}$ despite optimal statin treatment. ${ }^{14}$ Importantly, LDL-C reduction to $0.98 \mathrm{mmol} / \mathrm{L}$ in ODYSSEY-OUTCOMES and $0.78 \mathrm{mmol} / \mathrm{L}$ in FOURIER was found to be safe in both instances.

\section{Measuring inflammation and endothelial function}

Metabolically active inflammatory cells utilise more glucose than non-activated cells, and the degree of metabolic activity can be measured using FDG-PET/CT. ${ }^{16} 17$ Cellular FDG uptake is largely irreversible and correlates with glucose usage and macrophage numbers in both tumour cells and atherosclerotic plaques. ${ }^{18} 19$ FDG-PET imaging has been successfully used to determine culprit plaques responsible for transient ischaemic attacks and stroke, as well providing a potential independent prognostic marker for CV risk. ${ }^{2021}$ The signal has also been shown to be responsive to both anti-inflammatory and statin therapy, with clear reductions in FDG uptake noted in both human and animal models of atherosclerosis. $^{22} 23$ Taken together, these findings suggest a role for FDG PET/CT imaging in the assessment of the antiinflammatory potential of novel compounds such as PCSK9 inhibition therapies.

Endothelial function can be measured in the peripheral vasculature in a number of different ways. Flowmediated dilatation (FMD) of the brachial artery can be assessed non-invasively using ultrasound, to indicate nitric oxide (NO) dependent endothelial function. ${ }^{24}$ Impaired 
brachial artery FMD predicts CV events independently of established CV risk factors in patients with established atherosclerotic vascular disease. The degree of endothelial dysfunction is related to the extent of vascular oxidative stress and inflammation. ${ }^{25}$ Additionally, arterial stiffening is related to atherosclerotic and arteriosclerotic processes in the vessel wall. Increased stiffness of the central arteries is an independent predictor of CV outcomes in various patient groups as well as in general population, ${ }^{26}$ and can directly accelerate atherogenesis. ${ }^{27} 28$ It is regulated by numerous factors with mean arterial pressure and structural changes in the arterial wall components (ie, elastin and collagen), as well as ageing, considered as the main determinants. Pulse-wave velocity (PWV) and the aortic Augmentation Index (AIx) are measurable markers of arterial stiffness and related arterial properties. ${ }^{24}$

In the clinical setting, endothelial dysfunction is detected as an attenuated vasodilatory response to mechanical or pharmacological interventions that promote release of NO. ${ }^{29}$ Oxidation of LDL (oxLDL) is a key event in the reduction of $\mathrm{NO}$ production via its adverse effects on endothelial nitric oxide synthase (eNOS) production and on the destabilisation of eNOS mRNA. ${ }^{30}$ Elevations in C reactive protein, a common sequelae of the inflammatory cascade, is seen as a bystander biomarker of increased production of superoxide from nicotinamide adenosine dinucleotide phosphate oxidase via p38 kinase activation. ${ }^{31}$ Amplification of this circuit can further decrease the levels of active NO. In this context, it was suggested that both lipid-lowering (particularly with statins) and anti-inflammatory treatment have short-term and longterm benefits on endothelial function. ${ }^{632} 33$

\section{METHODS}

This is a single-centre, randomised, open label, parallel group, mechanistic physiological study. It will be run at the Vascular Research Clinics, Division of Experimental Medicine and Immunotherapeutics, University of Cambridge, Cambridge, UK. Patients will be recruited from local clinics, as well as from co-opted participant identification centres, and via study advertisements in the general press. Overall study coordination will be provided by the Cardiovascular Office of the Clinical Trials Unit, Cambridge University Hospitals (CUH) National Health Service (NHS) Foundation Trust. Data will be held in an anonymised centrally maintained database, with external quality control checks undertaken by rhe Clinical Trials Unit.

The study is sponsored by CUH NHS Foundation Trust and will adhere to the principles of the International Conference for Harmonisation of Technical Requirements for Registration of Pharmaceuticals for Human Use (ICH) Good Clinical Practice. The study received a favourable outcome from the Wales Research Ethics Committee 4 (REC reference: 17/WA/0285) and the Administration of Radioactive Substances Advisory Committee and was registered on clinicaltrials.gov prior to study commencement. The Medicines \& Healthcare products Regulatory Agency indicated that this study did not require a Clinical Trial Authorisation from the regulatory body. The results of this study will be reported through peer-reviewed journals and conference presentations.

\section{Study population}

The study will include 60 participants aged over 40 years with an established clinical diagnosis of stable CVD (ascertained by having either a previous diagnosis of myocardial infarction (MI), ischaemic stroke, peripheral vascular disease, evidence of occlusive coronary heart disease or a history of coronary, carotid or peripheral revascularisation). The inclusion and exclusion criteria are detailed in box 1. In summary, patients with a significant CV event within the prior 6 months to enrolment; uncontrolled type 2 diabetes mellitus; active malignancy; renal, hepatic thyroid or haematological dysfunction; and intolerance to statin therapy will be excluded from the study.

\section{Study protocol}

All potential participants will be given a patient information sheet and will have at least 24 hours to review prior to providing informed consent to the study team (see attached online supplemental file). Study procedures will only be conducted following formal written informed consent at the screening visit (V1). Baseline blood tests will include lipid profile (including LDL, HDL, triglycerides, lipoprotein(a), apolipoprotein A-I, and apolipoprotein B); electrolytes; renal, liver and thyroid functions; full blood count; glycated haemoglobin and a urinary pregnancy screen (where appropriate). Baseline vital signs, ECG, ascertainment of prior medical history and concomitant medications, as well as physical examination will be undertaken. Participants not already on a predefined high-intensity statin (as per protocol) will be prescribed as such and enter a run-in phase of 1 month (see box 2), after which their lipid profile will be rechecked to ensure eligibility (V1b). A run-in phase will not be required for participants already on suitable therapy, who will proceed directly to FDG-PET scanning.

The study schedule is detailed in figure 1. In brief, patients will undergo FDG-PET scanning of their aorta and carotid arteries at $\mathrm{V} 2$ prior to their randomisation to study treatment. At V3, all participants will be randomly allocated on a 1:1 basis by the study team using a centrally generated randomisation list, to either alirocumab $150 \mathrm{mg}$ subcutaneously every 2 weeks, or ezetimibe $10 \mathrm{mg}$ orally daily on top of their pre-existing high-intensity statin treatment. At the same visit, predose assessments of baseline endothelial function measurements by FMD and other vascular indices will be undertaken. Subsequently, study participants will attend every 2 weeks (V4-V6) for safety blood tests (renal, liver function, creatine kinase (CK)) and dosing. A tablet check will be undertaken where appropriate. A repeat FDG-PET/CT scan will be undertaken at V7, and endothelial function and vascular index measurements will be repeated at V8. Lipid profile, 


\section{Box 1 Inclusion and exclusion criteria for INTENSITY-HIGH}

\section{Inclusion criteria}

- Patients with high-risk cardiovascular disease (CVD) with lowdensity lipoprotein cholesterol (LDL-C) of $\leq 4.0 \mathrm{mmol}^{*}$ OR patients with very high-risk CVD with $L D L-C \leq 3.5 \mathrm{mmol} / \mathrm{L} . \dagger$

- Male or female patients over 40 years of age inclusive at screening, with a body weight $\geq 45 \mathrm{~kg}$ and BMl of $\geq 18$ to $\leq 40 \mathrm{~kg} / \mathrm{m}^{2}$.

- History of stable CVD, defined as previous myocardial infarction (MI) (STEMI or NSTEMI), angioplasty, documented Coronary Artery Disease (stress echo, CT coronary angiography or invasive angiography), stroke, transient ischaemic attack or peripheral vascular disease without a recent event in the last 6 months (ie, acute coronary syndrome, unstable angina, coronary artery bypass grafting (CABG), Percutaneous Coronary Intervention $(\mathrm{PCl})$, stroke, $\mathrm{MI}$ and carotid endarterectomy).

\section{Exclusion criteria}

- Uncontrolled hypertension blood pressure of $>180 / 110 \mathrm{~mm} \mathrm{Hg}$ on repeated measurements.

- Fasting hypertriglyceridaemia with fasting triglyceride of $>10 \mathrm{mmol} / \mathrm{L}$ at screening

- Pregnancy or combined contraceptive pill or hormone replacement therapy or childbearing potential.

- Any concomitant condition that, at the discretion of the investigator, may affect the participant's ability to complete the study.

- Any known sensitivity to alirocumab or monoclonal antibodies.

- Patients with history of hypersensitivity reactions to any of the study drugs.

- History of significant LFTs (3xupper limit of normal (ULN) alanine transaminase or aspartate transaminase elevation) by previous statin treatment.

- History of previous myositis associated with statin treatment (ie, myalgias or asymptomatic creatine kinase elevation $>5 \times$ ULN).

- Type 1 or type 2 diabetes, which is insulin dependent or on oral hypoglycaemics/diet with glycated haemoglobin $(\mathrm{HbA1c})>8 \%(\mathrm{OR}$ $\mathrm{HbA} 1 \mathrm{c}>64 \mathrm{mmol} / \mathrm{mol}$ ).

- History of any acute cardiovascular (CV) event within 6 months prior to the screening period.

- Rheumatoid arthritis, connective tissue disorders and other conditions known to be associated with active chronic inflammation (eg, inflammatory bowel disease).

- Untreated hypothyroidism, known autoimmune myositis.

- Patients with chronic kidney disease (defined as eGFR $<30 \mathrm{~mL}$ $\min / 1.73 \mathrm{~m}^{2}$ ) at baseline will be excluded from the study.

- Participant in a previous research study in the last 3 years which involved exposure to significant ionising radiation (ie, cumulative research radiation dose $>5 \mathrm{mSv}$ ).

- History of malignancy within the past 5 years (with the exception of localised carcinoma of the skin that has been resected for cure).

- History of alcohol/drug abuse or dependence within 6 months of the study.

- Use of systemic corticosteroids at the time of scanning.

- Lack of ability to provide informed consent.

Treatment with medications that result in significant drug-drug interactions with study medications.

*High risk is defined as a history of any of the following: acute coronary syndrome, coronary or other arterial revascularisation procedures, chronic heart disease, ischaemic stroke and peripheral arterial disease.

\section{Box 1 Continued}

†Very high risk is defined as recurrent CV events or CV events in more than one vascular bed (ie, polyvascular disease).

oxLDL and high-sensitivity CRP (hsCRP) will be assessed at V3, V4 and V8. The total duration of therapy from V3 to $\mathrm{V} 8$ will be 8 weeks.

Withdrawal criteria have been included to maintain the safety/risk benefit of the study, such as liver function derangement (eg, rise in alanine transaminase or aspartate transaminase to three times the upper limit of normal (ULN)) and evidence of myopathy (detected by a rise in CK to five times the ULN). Serious adverse events will be reported to the sponsor as per ICH Good Clinical Practice guidelines.

\section{Vascular assessments}

\section{FMD and glyceryl trinitrate (GTN)-mediated dilatation}

FMD will be measured using using a high-resolution ultrasound scanner with a $10 \mathrm{MHz}$ linear-array transducer (MyLab25 Gold Ultrasound Machine, Esaote, Italy), with the volunteer resting in the supine position for at least $5 \mathrm{~min}$. The brachial artery in the right arm will be identified $5-10 \mathrm{~cm}$ above the antecubital fossa using ultrasound. FMD will be measured as described previously, using Cardiovascular Suite software (Quipu srl, Pisa, Italy) ${ }^{34}$

GTN-mediated dilation will be measured using the same equipment, in the same artery. GTN (25 mcg) will be administered sublingually, with the brachial artery diameter measured at baseline and $5 \mathrm{~min}$ after GTN, as described. ${ }^{34}$

\section{Arterial stiffness and central haemodynamics}

Central haemodynamics as well as arterial stiffness, assessed by determination of the AIx and the carotid-tofemoral PWV, will be determined using a commercially available SphygmoCor system (AtCor Medical, Sydney, Australia), as previously described. ${ }^{35}$

\section{Carotid Intima-Media Thickness (IMT)}

Carotid IMT will be measured by using a high-resolution ultrasound scanner with a $10 \mathrm{MHz}$ linear-array transducer (MyLab25 Gold Ultrasound Machine, Esaote, Italy), with the volunteer resting in the supine position for at least 5 minutes. The common carotid artery will be identified and scanned, $2 \mathrm{~cm}$ below its bifurcation. Measurements will be repeated three times, and the average of the values will be recorded.

Box 2 List of high intensity statin regimens for the INTENSITY-HIGH protocol

Simvastatin $80 \mathrm{mg}$ once daily.

Atorvastatin $40-80 \mathrm{mg}$ once daily.

Rosuvastatin $20-40 \mathrm{mg}$ once daily. 


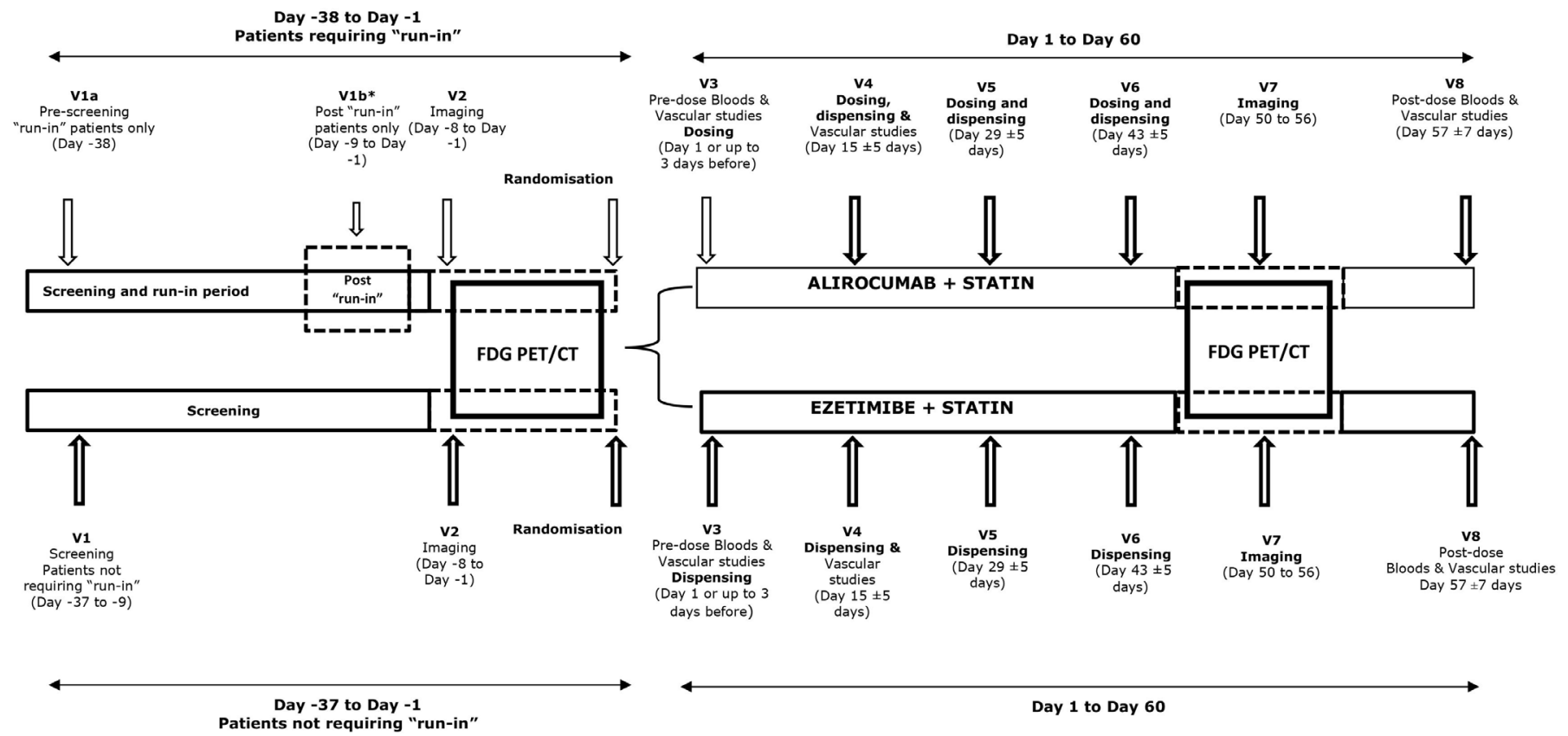

Figure 1 Synopsis of the study design. After a screening visit (V1), all eligible volunteers will attend V2 for an FDG PET/CT scan. They will then attend $\mathrm{V} 3$, in which they will have predose blood tests and vascular studies, and then will be randomised to either single subcutaneous dose of alirocumab $150 \mathrm{mg}$ or ezetimibe $10 \mathrm{mg}$ once per day. They will then attend three subsequent dosing visits (V4-V6) and undergo a repeat FDG PET/CT scan after 8 weeks of therapy. A final set of vascular studies will be undertaken at V8. FDG PET/CT, 18-fluorodeoxyglucose positron emission tomography/CT; V1, visit 1; V2, visit 2; V3, visit 3; V4, visit 4; V5, visit 5; V6, visit 6; V7, visit 7; V8, visit 8.

\section{Outcome measures}

The primary outcome will be the change in vascular inflammation on FDG-PET /CT using derivatives of standard uptake value and tissue to background ratio (TBR) in the aorta and carotid arteries of patients following approximately 8 weeks of treatment.

A variety of secondary outcome measures include comparison of the effects of adding alirocumab or ezetimibe on top of high-dose statin treatment on FMD and sublingual GTN response, as surrogate measures of endothelial-dependent and endothelial-independent vasodilation, respectively, changes in Aix, PWV, cIMT, lipid profile, hsCRP and other markers of systemic inflammation. An assessment of safety and tolerability will be undertaken as part of standard clinical practice, including physical examination, blood pressure, heart rate, 12-lead ECGs, clinical laboratory tests and adverse event reporting.

\section{Image analysis}

FDG-PET/CT imaging of the aorta and carotid arteries will be performed at V2 and V7. The images will be analysed using OsiriX imaging software (Pixmeo, Bernex, Switzerland). The maximum standardised uptake value of FDG within each axial PET/CT fused image of artery, containing wall and lumen, will be divided by the average blood FDG concentration in the superior vena cava (for aorta) or jugular vein (for carotids) to yield an arterial maximum TBR, as a quantitative measure of arterial tracer uptake corrected for systemic uptake. Average TBR for ascending, descending and abdominal aorta, as well as average TBR for the right and left carotid arteries combined, will be calculated. Additional analysis will examine the proportion of aortic slices with a TBR of $>1.6$, which is used as a threshold to define highly inflamed areas of aorta. Imaging data will be analysed blinded to the allocated study treatment and visit number.

\section{Statistical methods}

For each cross-sectional slice of each vessel on the FDGPET/CT scans, mean and maximum SUV and TBR values will be calculated. The vessel with the highest average maximum TBR will be defined as the index vessel. The change from baseline in average maximum TBR in the index vessel will be analysed using analysis of covariance, estimating the treatment effect, and including the baseline value as a covariate. Point estimates and corresponding 95\% CIs will be reported.

The sample size estimation for this study assumes a $15 \%$ change in TBR of FDG uptake, in keeping with the predicted change in LDL-C and effects on TBR seen in previous cholesterol-lowering studies. ${ }^{23}$ To achieve $90 \%$ power with a significance level of $5 \%$, it is estimated that 30 subjects per group will be required.

\section{Patient and public involvement}

Lay members of the ethics committee reviewed this study and made constructive comments, which have been addressed. Patients were not involved in the recruitment to or conduct of the study. All patients provided full informed consent, with at least 24 hours to consider the information and discuss the trial in detail with the 
investigators. The results of the study will be disseminated to study patients at the end of the trial.

\section{SUMMARY}

The current mainstay of medical treatment of CVD focusses on primary and secondary prevention. However, it is still unclear what the lowest threshold of total and LDL-C should be to attain significant reductions in CV risk in patients with established CVD. The aim of this research study was to better define the relationship between inflammation and LDL-C modification, especially at lower LDL-C levels in high-risk patients combining established and novel LDL-lowering therapies. It is envisaged these mechanistic data from INTENSITY-HIGH will be useful in adding to the armamentarium of knowledge supporting lipid lowering in patients with established CVD.

\section{ETHICS AND DISSEMINATION}

The study received a favourable opinion from the Wales Research Ethics Committee 4, was registered on clinicaltrials.gov and conformed to ICH Good Clinical Practice. The results of this study will be reported through peerreviewed journals and conference presentations.

\section{Author affiliations}

${ }^{1}$ Division of Experimental Medicine and Immunotherapeutics (EMIT), University of Cambridge, Cambridge, UK

${ }^{2}$ Lipid Clinic, Cambridge University Hospitals NHS Foundation Trust, Cambridge, UK ${ }^{3}$ Cambridge Clinical Trials Unit, Cambridge University Hospitals NHS Foundation Trust, Cambridge, UK

\section{Twitter Paul J Cacciottolo @pawlu}

Acknowledgements This INTENSITY-HIGH study is funded by the Jon Moulton Charitable Trust and will be conducted at the NIHR Cambridge Clinical Research Facility. The funder had no role in the conception, design and conduct of the study. The authors acknowledge the significant support of the National Institute for Health Research (NIHR) Cambridge Biomedical Research Centre and the NIHR Clinical Research Network, as well as the Core Biochemical Assay Laboratory, Cambridge. The views expressed are those of the authors and not necessarily those of the NHS, the NIHR or the Department of Health and Social Care.

Contributors PJC, MSK and JC drafted the manuscript. JC, CMM and IBW reviewed and edited the manuscript. PJC, MSK, EHS, HP, FK, EV, JH, AH, CMM, IBW and JC contributed to its scientific content.

Funding The INTENSITY-HIGH study is funded by the Jon Moulton Charitable Trust, with additional funds from the Addenbrookes Charitable Trust. Grant numbers are not applicable. Setup and running of the study and decision to publish results is independent of the funders.

Competing interests None declared.

Patient consent for publication Not required.

Provenance and peer review Not commissioned; externally peer reviewed.

Supplemental material This content has been supplied by the author(s). It has not been vetted by BMJ Publishing Group Limited (BMJ) and may not have been peer-reviewed. Any opinions or recommendations discussed are solely those of the author(s) and are not endorsed by BMJ. BMJ disclaims all liability and responsibility arising from any reliance placed on the content. Where the content includes any translated material, BMJ does not warrant the accuracy and reliability of the translations (including but not limited to local regulations, clinical guidelines, terminology, drug names and drug dosages), and is not responsible for any error and/or omissions arising from translation and adaptation or otherwise.
Open access This is an open access article distributed in accordance with the Creative Commons Attribution Non Commercial (CC BY-NC 4.0) license, which permits others to distribute, remix, adapt, build upon this work non-commercially, and license their derivative works on different terms, provided the original work is properly cited, appropriate credit is given, any changes made indicated, and the use is non-commercial. See: http://creativecommons.org/licenses/by-nc/4.0/.

ORCID iDs

Paul J Cacciottolo http://orcid.org/0000-0001-8780-5685

Michalis S Kostapanos http://orcid.org/0000-0002-7513-5319

Joseph Cheriyan http://orcid.org/0000-0001-6921-1592

\section{REFERENCES}

1 Libby P. Inflammation in atherosclerosis. Nature 2002;420:868-74.

2 Sharrett AR, Ballantyne CM, Coady SA, et al. Coronary heart disease prediction from lipoprotein cholesterol levels, triglycerides, lipoprotein(a), apolipoproteins A-I and B, and HDL density subfractions: The Atherosclerosis Risk in Communities (ARIC) Study. Circulation 2001;104:1108-13.

3 Cholesterol Treatment Trialists' (CTT) Collaborators, Mihaylova B, Emberson J, et al. The effects of lowering LDL cholesterol with statin therapy in people at low risk of vascular disease: meta-analysis of individual data from 27 randomised trials. Lancet 2012;380:581-90.

4 Oesterle A, Laufs U, Liao JK. Pleiotropic effects of statins on the cardiovascular system. Circ Res 2017;120:229-43.

5 Kostapanos MS, Milionis HJ, Elisaf MS. An overview of the extra-lipid effects of rosuvastatin. $J$ Cardiovasc Pharmacol Ther 2008:13:157-74.

6 Mäki-Petäjä KM, Booth AD, Hall FC, et al. Ezetimibe and simvastatin reduce inflammation, disease activity, and aortic stiffness and improve endothelial function in rheumatoid arthritis. J Am Coll Cardiol 2007;50:852-8.

7 Lind L, Berglund L, Larsson A, et al. Endothelial function in resistance and conduit arteries and 5-year risk of cardiovascular disease. Circulation 2011;123:1545-51.

8 Hingorani AD, Cross J, Kharbanda RK, et al. Acute systemic inflammation impairs endothelium-dependent dilatation in humans. Circulation 2000;102:994-9.

9 Lee $\mathrm{KH}$, Jeong $\mathrm{MH}$, Kim HM, et al. Benefit of early statin therapy in patients with acute myocardial infarction who have extremely low low-density lipoprotein cholesterol. J Am Coll Cardiol 2011;58:1664-71.

10 Cannon CP, Blazing MA, Giugliano RP, et al. Ezetimibe added to statin therapy after acute coronary syndromes. $N$ Engl J Med 2015;372:2387-97

11 Sabatine MS, Wiviott SD, Im K, et al. Efficacy and safety of further lowering of low-density lipoprotein cholesterol in patients starting with very low levels: a meta-analysis. JAMA Cardiol 2018;3:823-8.

12 Mach F, Baigent C, Catapano AL. ESC/EAS guidelines for the management of dyslipidaemias: lipid modification to reduce cardiovascular risk. Eur Heart J. August 2019;2019.

13 Kostapanos MS, Cacciottolo PJ, Hubsch A, et al. Investigating the lowest threshold of vascular benefits from LDL cholesterol lowering with a PCSK9 mAb inhibitor (alirocumab) in healthy volunteers - a mechanistic physiological study (INTENSITY-LOW): protocol and study rationale. J Drug Assess 2019;8:167-74.

14 Sabatine MS, Giugliano RP, Keech AC, et al. Evolocumab and clinical outcomes in patients with cardiovascular disease. N Engl J Med 2017;376:1713-22.

15 Schwartz GG, Steg PG, Szarek M, et al. Alirocumab and cardiovascular outcomes after acute coronary syndrome. $N$ Engl $J$ Med 2018;379:2097-107.

16 Kubota R, Yamada S, Kubota K, et al. Intratumoral distribution of fluorine-18-fluorodeoxyglucose in vivo: high accumulation in macrophages and granulation tissues studied by microautoradiography. J Nucl Med 1992;33:1972-80.

17 Tawakol A, Migrino RQ, Bashian GG, et al. In vivo 18Ffluorodeoxyglucose positron emission tomography imaging provides a noninvasive measure of carotid plaque inflammation in patients. $J$ Am Coll Cardiol 2006;48:1818-24.

18 Rudd JHF, Myers KS, Bansilal S, et al. 18Fluorodeoxyglucose positron emission tomography imaging of atherosclerotic plaque inflammation is highly reproducible. J Am Coll Cardiol 2007:50:892-6.

19 Rudd JHF, Warburton EA, Fryer TD, et al. Imaging atherosclerotic plaque inflammation with [18F]-fluorodeoxyglucose positron emission tomography. Circulation 2002;105:2708-11. 
20 Davies JR, Rudd JHF, Fryer TD, et al. Identification of culprit lesions after transient ischemic attack by combined $18 \mathrm{~F}$ fluorodeoxyglucose positron-emission tomography and high-resolution magnetic resonance imaging. Stroke 2005;36:2642-7.

21 Figueroa AL, Abdelbaky A, Truong QA, et al. Measurement of arterial activity on routine FDG PET/CT images improves prediction of risk of future cv events. JACC Cardiovasc Imaging 2013:6:1250-9.

22 Ogawa M, Magata Y, Kato T, et al. Application of 18F-FDG PET for monitoring the therapeutic effect of antiinflammatory drugs on stabilization of vulnerable atherosclerotic plaques. $J \mathrm{Nucl} \mathrm{Med}$ 2006;47:1845-50.

$23 \mathrm{Wu}$ Y-W, Kao H-L, Huang C-L, et al. The effects of 3-month atorvastatin therapy on arterial inflammation, calcification, abdominal adipose tissue and circulating biomarkers. Eur J Nucl Med Mol Imaging 2012;39:399-407.

24 Sun ZDY, Cheriyan J. Non-invasive measurements of arterial function: what? when? why should we use them? Heart 2019;105:1203-11.

25 Green DJ, Jones H, Thijssen D, et al. Flow-Mediated dilation and cardiovascular event prediction does nitric oxide matter? 2011.

26 Ben-Shlomo Y, Spears M, Boustred C, et al. Aortic pulse wave velocity improves cardiovascular event prediction: an individual participant meta-analysis of prospective observational data from 17,635 subjects. J Am Coll Cardiol 2014;63:636-46.

27 McEniery CM, Cockcroft JR. Does arterial stiffness predict atherosclerotic coronary events? Adv Cardiol 2007;44:160-72.
28 Mattace-Raso FUS, van der Cammen TJM, Hofman A, et al Arterial stiffness and risk of coronary heart disease and stroke: the Rotterdam study. Circulation 2006;113:657-63.

29 Deanfield JE, Halcox JP, Rabelink TJ. Endothelial function and dysfunction: testing and clinical relevance. Circulation 2007:115:1285-95

30 Kinlay S, Libby P, Ganz P. Endothelial function and coronary artery disease. Curr Opin Lipidol 2001;12:383-9.

31 Frey RS, Ushio-Fukai M, Malik AB. Nadph oxidase-dependent signaling in endothelial cells: role in physiology and pathophysiology. Antioxid Redox Signal 2009;11:791-810.

32 Mäki-Petäjä KM, Elkhawad M, Cheriyan J, et al. Anti-Tumor necrosis factor- $\alpha$ therapy reduces aortic inflammation and stiffness in patients with rheumatoid arthritis. Circulation 2012;126:2473-80.

33 Dupuis J, Tardif JC, Cernacek P, et al. Cholesterol reduction rapidly improves endothelial function after acute coronary syndromes. The RECIFE (reduction of cholesterol in ischemia and function of the endothelium) trial. Circulation 1999;99:3227-33.

34 Gori T, Muxel S, Damaske A, et al. Endothelial function assessment: flow-mediated dilation and constriction provide different and complementary information on the presence of coronary artery disease. Eur Heart J 2012;33:363-71.

35 Lind L. Relationships between three different tests to evaluate endothelium-dependent vasodilation and cardiovascular risk in a middle-aged sample. J Hypertens 2013;31:1570-4. 